\title{
Effect of substrate elasticity on evaporation kinetics and evaporative deposition of aqueous polystyrene nanoparticles droplets
}

\author{
Ying-Song Yu ${ }^{1 *}$, Yi-Qi Zhu ${ }^{1}$, XianFu Huang ${ }^{2}$, Jin-Zhi Zhou ${ }^{1 *}$, and An Zhou ${ }^{1}$ \\ ${ }^{1}$ Department of Mechanics, School of Civil Engineering, Architecture and Environment, Hubei University of Technology, \\ Wuhan 430068, China; \\ ${ }^{2}$ State Key Laboratory of Nonlinear Mechanics, Institute of Mechanics, Chinese Academy of Sciences, Beijing 100190, China
}

Received February 1, 2020; accepted June 3, 2020; published online September 2, 2020

\begin{abstract}
Evaporation of aqueous polystyrene (PS) nanoparticles droplets on silicon and polydimethylsiloxane (PDMS) surfaces was studied. Experimental results showed that softer PDMS surfaces yielded a longer constant contact radius (CCR) stage, which could be ascribed to surface deformation of PDMS induced by the vertical component of liquid-vapor interfacial tension. Ringlike depositions of nanoparticles with different crack patterns were found on both silicon and PDMS surfaces. In-situ observation of crack formation showed that nanoparticle movement on the silicon surface was impeded, resulting in radial cracks with periodic distribution. In contrast, nanoparticles were shown to move easily on the PDMS surface. This observation indicated the difference in crack patterns on surfaces could be attributed to the friction force between nanoparticles and the substrate. A large friction force between nanoparticles and the substrate prevented cracks from moving, resulting in a radial crack pattern with periodic distribution, while a small friction force produced multiple large cracks.
\end{abstract}

droplet, evaporation, nanoparticle, constant contact radius stage, crack

PACS number(s): $61.30 . \mathrm{Hn}, 61.46 . \mathrm{Fg}, 68.03 . \mathrm{Fg}$

Citation: Y.-S. Yu, Y.-Q. Zhu, X. F. Huang, J.-Z. Zhou, and A. Zhou, Effect of substrate elasticity on evaporation kinetics and evaporative deposition of aqueous polystyrene nanoparticles droplets, Sci. China-Phys. Mech. Astron. 63, 114612 (2020), https://doi.org/10.1007/s11433-020-1589-1

\section{Introduction}

Evaporation-induced deposition has broad applications in fields such as ink-jet printing, photonic crystal fabrication, functional materials, DNA/RNA mapping, and disease diagnosis [1-8]. Extensive studies have shown particle size and geometry [9-11], physical and chemical properties of the substrate [10,12-14] and liquid [15-18], and environmental conditions [19-21] play important roles in evaporative de-

*Corresponding authors (Ying-Song Yu, email: yuys@hbut.edu.cn; Jin-Zhi Zhou, email: zhoujz@hbut.edu.cn) position. There are at least two issues limiting the formation of uniform or ordered evaporative deposition. The first is the coffee-ring effect [22], which is widely accepted to be the result of contact line pinning [22] and suppression of Marangoni flow [23]. The second is the formation of cracks. When a liquid containing nanoparticles [24-27] or biological materials including proteins [28,29] and blood [30] evaporates completely on a solid surface, cracks with different morphologies such as parallel patterns [31], circular cracks [32], wavy cracks [29,33], radial cracks [34], craquelures, as well as spiral cracks [35] are typically formed. The formation of cracks has been found to be induced by increased stress 
due to capillary forces [33]. Experiments have shown the formation and propagation of cracks are dependent on parameters including particle size, solvent, substrate, environmental conditions, as well as film thickness. Cracking has an adverse effect on the applications of evaporative deposition in fields of coating and ink-jet printing and so on. In order to improve evaporative deposition applications, it is necessary to eliminate cracking. In 2013, Jin et al. [36] found that cracking can be avoided if enough emulsion droplets are added into a suspension.

Unlike hard substrates with Young's modulus on the order of $100 \mathrm{GPa}$ or higher, a soft substrate like polydimethylsiloxane (PDMS), whose modulus is of the order of $1 \mathrm{MPa}$ or less depending on the mass ratio of the base to curing agent, has wide applications in lab-on-a-chip and microfluidic devices, etc. Relatively large surface deformation in thin films made of PDMS has been demonstrated to result from the vertical component of liquid-vapor interfacial tension [37-40]. This deformation has been shown to influence surface wettability [41] and droplet evaporation [4245]. During recent years, evaporative deposition of micro/ nanoparticles on PDMS surface has been studied extensively $[11,45-48]$ and substrate elasticity has been found to greatly influence evaporative deposition $[45,48]$.

In this paper, evaporation of aqueous PS nanoparticles droplets on clean silicon and PDMS surfaces was studied. Radial cracks with periodic distribution were found on the clean silicon surface. On PDMS surfaces, quantities of cracks and crack size were shown to depend on the mass ratio of the base to curing agent. In-situ observations of the top view using a digital microscope revealed the friction force between the substrate and the nanoparticles controlled the crack pattern.

\section{Experimental section}

Both silicon and PDMS with base-to-curing agent mass ratios of 5:1, 10:1 and 20:1 were used as substrates for studying evaporation of aqueous PS nanoparticles droplets. Polished silicon was ultrasonically stirred in ethanol and acetone, sequentially, for $10 \mathrm{~min}$ to ensure the surface was clean. Well-mixed PDMS was poured onto the silicon surface and spin-coated at $500 \mathrm{r} / \mathrm{min}$ (revolutions per minute) for $30 \mathrm{~s}$ and $2000 \mathrm{r} / \mathrm{min}$ for $30 \mathrm{~s}$, consecutively. The PDMS was solidified at $90^{\circ} \mathrm{C}$ for $3 \mathrm{~h}$. The thickness of each PDMS film was approx. $20 \mu \mathrm{m}$ [49].

An aqueous suspension of $10.13 \mathrm{wt} \%$ PS nanoparticles (PS02N, mean diameter: $42 \mathrm{~nm}$, Bangs Laboratories, USA) was diluted to a particle concentration of $0.64 \mathrm{wt} \%$. The diluted suspension was ultrasonically stirred for $15 \mathrm{~min}$ to ensure homogeneous distribution of PS nanoparticles. Aqueous suspension with a nominal volume of $0.6 \mu \mathrm{L}$ was ex- tracted using a micropipette and deposited on the PDMS and silicon surfaces. A droplet shape analyzer (Krüss DSA30, Germany) was adjusted to record the droplet as soon as possible at $1 \mathrm{fps}$ (frame per second), as shown in Figure 1. Droplet evaporation was also recorded from the top view using a digital microscope (Hirox KH-8700, Japan) at time intervals of 1 or $2 \mathrm{~s}$. The ambient temperature and relative humidity were $23^{\circ} \mathrm{C} \pm 1{ }^{\circ} \mathrm{C}$ and $40 \% \pm 2 \%$, respectively. No heat source was present and the room was closed as soon as possible to ensure that experimental conditions were nearly constant.

\section{Results and discussion}

\subsection{Evaporation of aqueous PS nanoparticles droplets on silicon surface}

Figure 2 shows the evolution of contact diameter and contact angle of an aqueous PS nanoparticles droplet on a silicon surface versus normalized time $\left(t_{\mathrm{f}}\right.$ is the total evaporation time). Evaporation started with the CCR mode due to contact angle hysteresis. The initial contact angle was approx. $76^{\circ}$ and the evaporation flux at the contact line was of singularity, indicating a lot of water molecules would diffuse from the edge to the environment. To keep the contact line pinned, there existed a strong capillary compensation flow inside the droplet towards the contact line. Such a flow would continuously carry nanoparticles towards the edge and the accumulation of nanoparticles at the contact line provided an

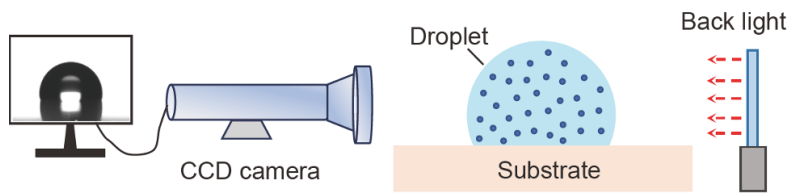

Figure 1 (Color online) Schematics of experimental setup.

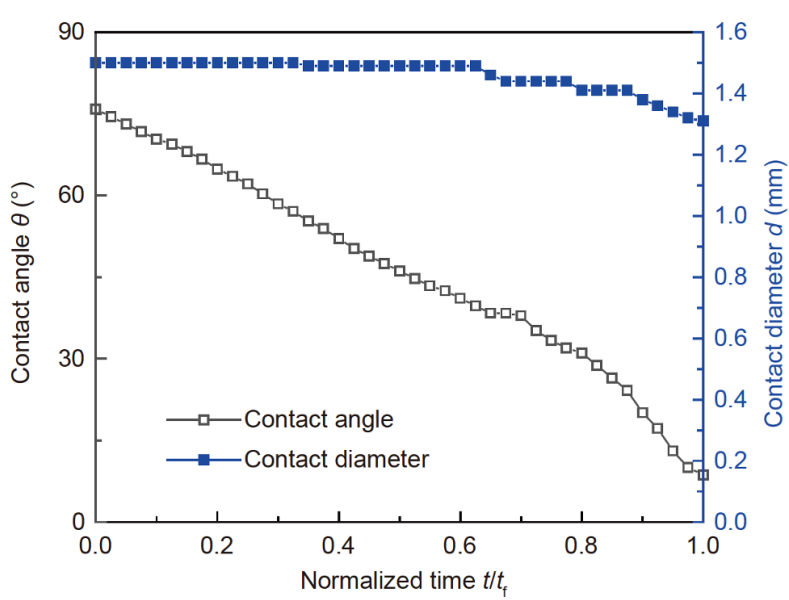

Figure 2 (Color online) Evaporation curve of an aqueous PS nanoparticles droplet on silicon surface. 
excess force to prevent the contact line from depinning. This could also be found from the top view images shown in Figure 3. At about $600 \mathrm{~s}$, a circular ring was visible at the edge of the droplet. When water evaporated completely, radial cracks with periodic distribution were found in the evaporative deposition, as shown in Figure 4.

The microscope was adjusted to record the evaporation of an aqueous droplet from the top view at a time interval of $1 \mathrm{~s}$, in order to gain a better understanding of the formation of the crack pattern. Figure 5 shows the short period when periodic cracks were formed. At $585 \mathrm{~s}$, a circular structure covered with a thin liquid film was visible at the edge of the droplet. As the thin film evaporated, the contact line receded from inside the pattern towards the edge. At $587 \mathrm{~s}$, several radial cracks were observed simultaneously, and soon afterwards more cracks were found. Meanwhile, liquid bridges were formed with these cracks. Since there was only a very thin water film covering the clusters of nanoparticles, liquid bridges could be broken very quickly and the clusters of nanoparticles experienced the horizontal component of the capillary force. Then a hoop stress was induced inside the clusters, however the strong friction force between the nanoparticles and silicon acted as a barrier to prevent the clusters from moving. Hence, all of the crack openings were very small and radical cracks with periodical distribution were formed as water evaporated quickly.

\subsection{Evaporation of aqueous PS nanoparticles droplets on PDMS surfaces}

Figure 6 shows the evolution of contact diameter and contact angle of aqueous PS nanoparticles droplets on PDMS surfaces with different mass ratios. Previous studies [42,43] have shown substrate elasticity influences the evaporation of pure water droplets, wherein longer CCR stages are found on the softer substrate due to the vertical component of liquidvapor interfacial tension. A similar phenomenon was also found in the case of aqueous PS nanoparticles droplets on PDMS substrates. Due to the absence of a singularity or weak singularity of evaporation flux at the contact line (the contact angles in the CCR stage were in the range of approx. $105^{\circ}$ to $80^{\circ}$ during evaporation), the internal flow was different from that of hydrophilic surfaces. It has been demonstrated that a symmetric counter-rotating vortex pair is found inside an evaporating droplet on a hydrophobic surface [50]. This flow will carry nanoparticles towards the contact line, however it is not easy for these particles to stay at the contact line due to Brownian motion.

Moreover, the slip condition of the contact line of evaporating droplets on PDMS surfaces behaves differently, resulting in a difference in internal flow. When a relatively large amount of nanoparticles are present at the contact line, the contact line will be pinned for a longer time due to the

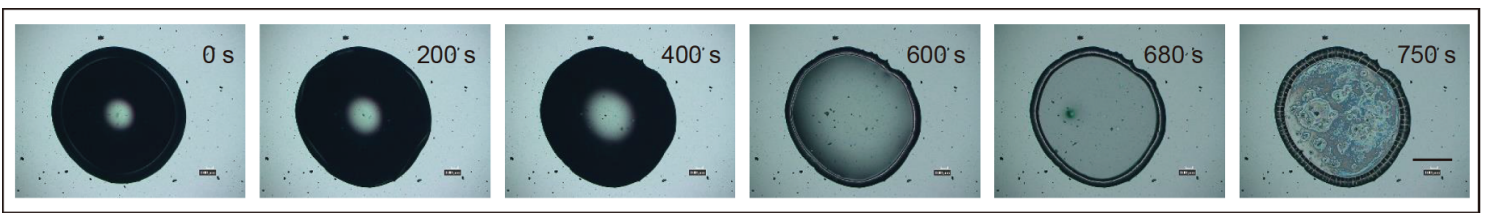

Figure 3 (Color online) Images of an evaporating aqueous PS nanoparticles droplet on silicon surface. Scale bar: $500 \mu \mathrm{m}$.
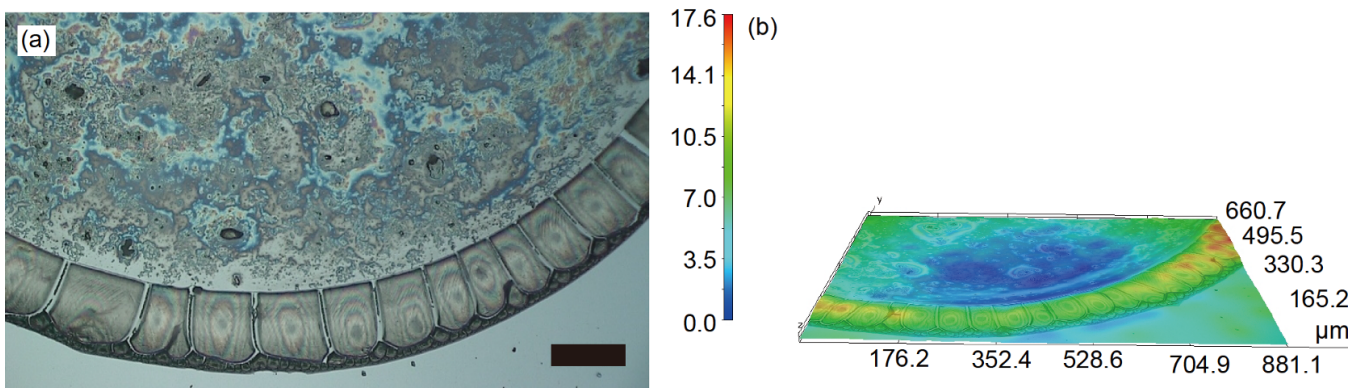

Figure 4 (Color online) Crack pattern of PS nanoparticles on silicon surface. (a) Magnification view (scale bar: $100 \mu \mathrm{m}$ ); (b) deposition height.

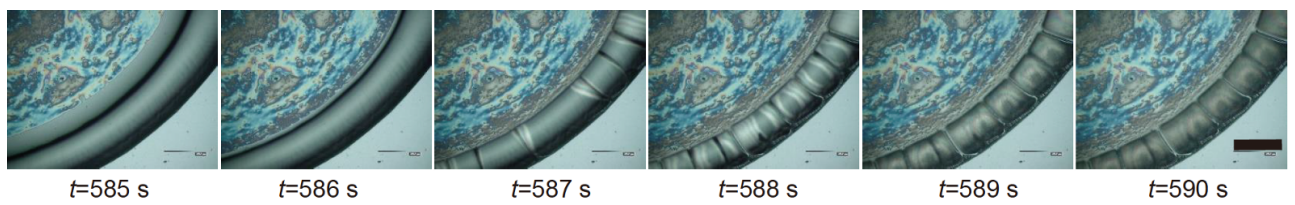

Figure 5 (Color online) Evolution of crack pattern of PS nanoparticles on silicon surface. Scale bar: $100 \mu \mathrm{m}$. 

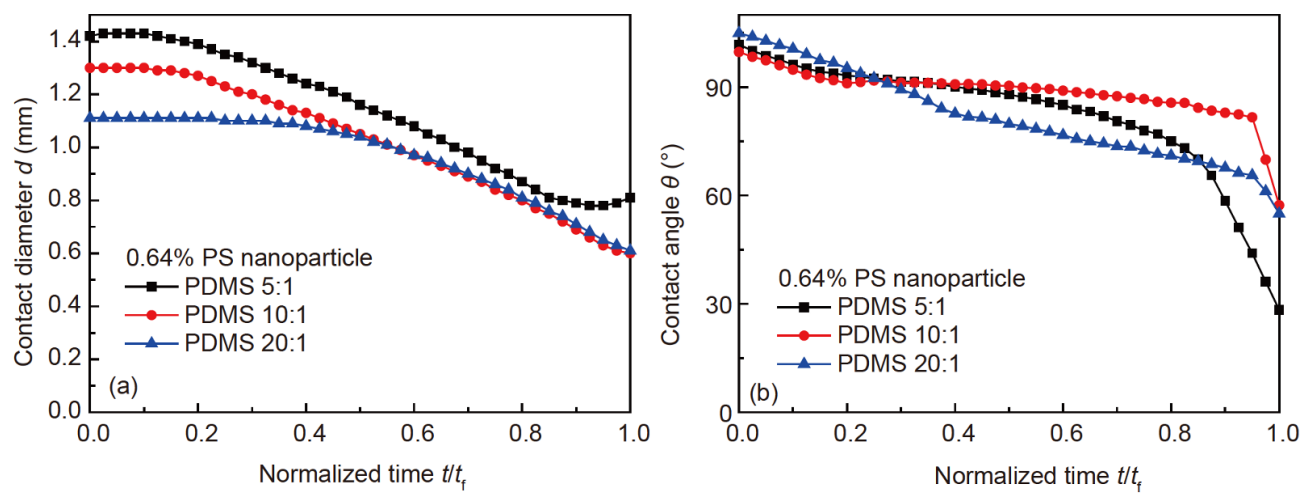

Figure 6 (Color online) Evaporation curves of aqueous PS nanoparticles droplets on PDMS surfaces. (a) Contact diameter; (b) contact angle.

additional contribution of these particles to adhesion. As the contact angle continuously decreases, the horizontal component of the capillary force acting on the nanoparticles at the contact line will increase. When the capillary force is greater than the total resistance force, the nanoparticles move inward. Moreover, from the images shown in Figure 7, it was found that the nanoparticles at the contact line receded with the receding contact line, indicating the friction force between nanoparticles and the PDMS surfaces was smaller than the horizontal component of the capillary force at this stage. As the droplet shrank, these nanoparticles would no longer recede due to the increase in the friction force between nanoparticles and PDMS. Coffee rings with one to several large cracks were found inside the initially wetted area with only a small amount of nanoparticles inside the ring. In addition, the quantity of large cracks seemed to be dependent on the substrate elasticity. More cracks were found on PDMS 5:1 and PDMS 10:1, while only one crack was formed on PDMS 20:1, as shown in Figure 7.

Figures 7 and 8 show ring-like depositions are easily observed inside aqueous droplets during evaporation. Figure 9 shows the evolution of crack patterns on PDMS surfaces. It was found that as the thin liquid film evaporated from the surface of the upper nanoparticles, cracks were likely to be formed. On PDMS 5:1, the opening between cracks was found to be larger than on the silicon surface. As the mass ratio increased to $10: 1$, the crack openings increased. On PDMS 20:1, no crack was observed in the local view (as shown in Figure 7(c), there was only one crack on PDMS 20:1 and hence it was difficult to predict where the crack might be formed). This observation indicated that the friction force between surface nanoparticles and the PDMS substrate depended on the mass ratio of base to curing agent.

As shown in Figures 5 and 9, there were thin films of water covering nanoparticle clusters. Hence it could be regarded that the nanoparticles made contact with the substrate and then there was a friction force acting on these nanoparticles. It could also be found in Figures 5 and 9 that as water continuously evaporated, some part of the evaporative deposition was lacking water on the upper surface, resulting in the formation of liquid bridges. As water evaporated, the liquid bridges broke suddenly and the lateral component of capillary force acted on both sides of the deposition. Under the action of capillary force, the clusters of nanoparticles experienced hoop stress resulting in strain. If the friction force was not large enough to overcome the lateral component of
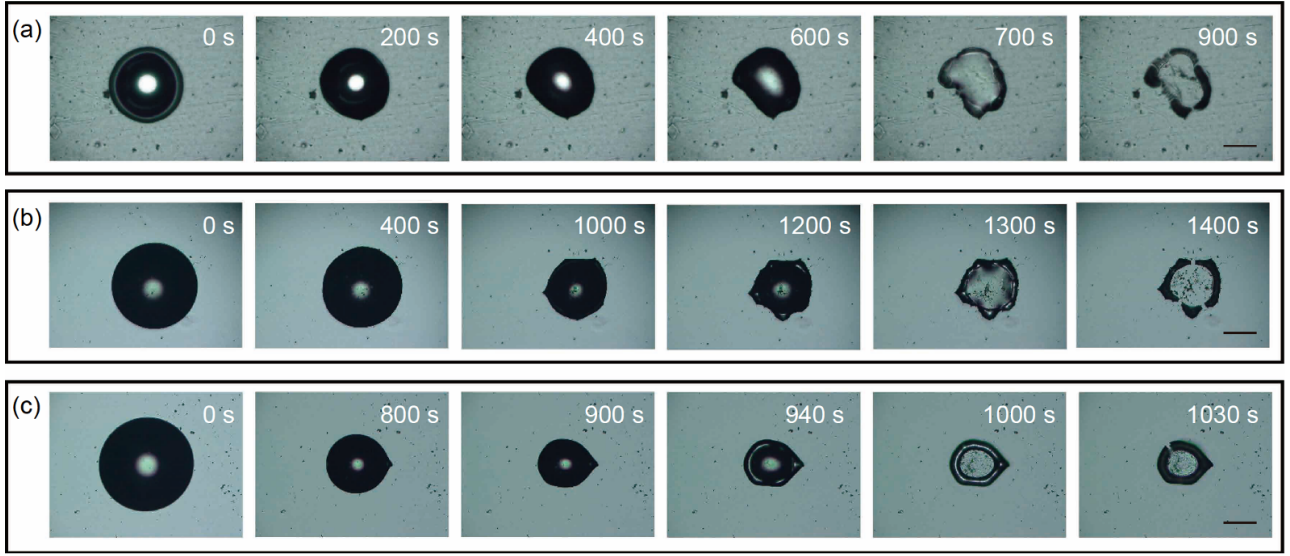

Figure 7 (Color online) Images of evaporating aqueous PS nanoparticles droplets on PDMS surfaces. (a) PDMS 5:1; (b) PDMS 10:1; (c) PDMS 20:1. Scale bars: $500 \mu \mathrm{m}$. 

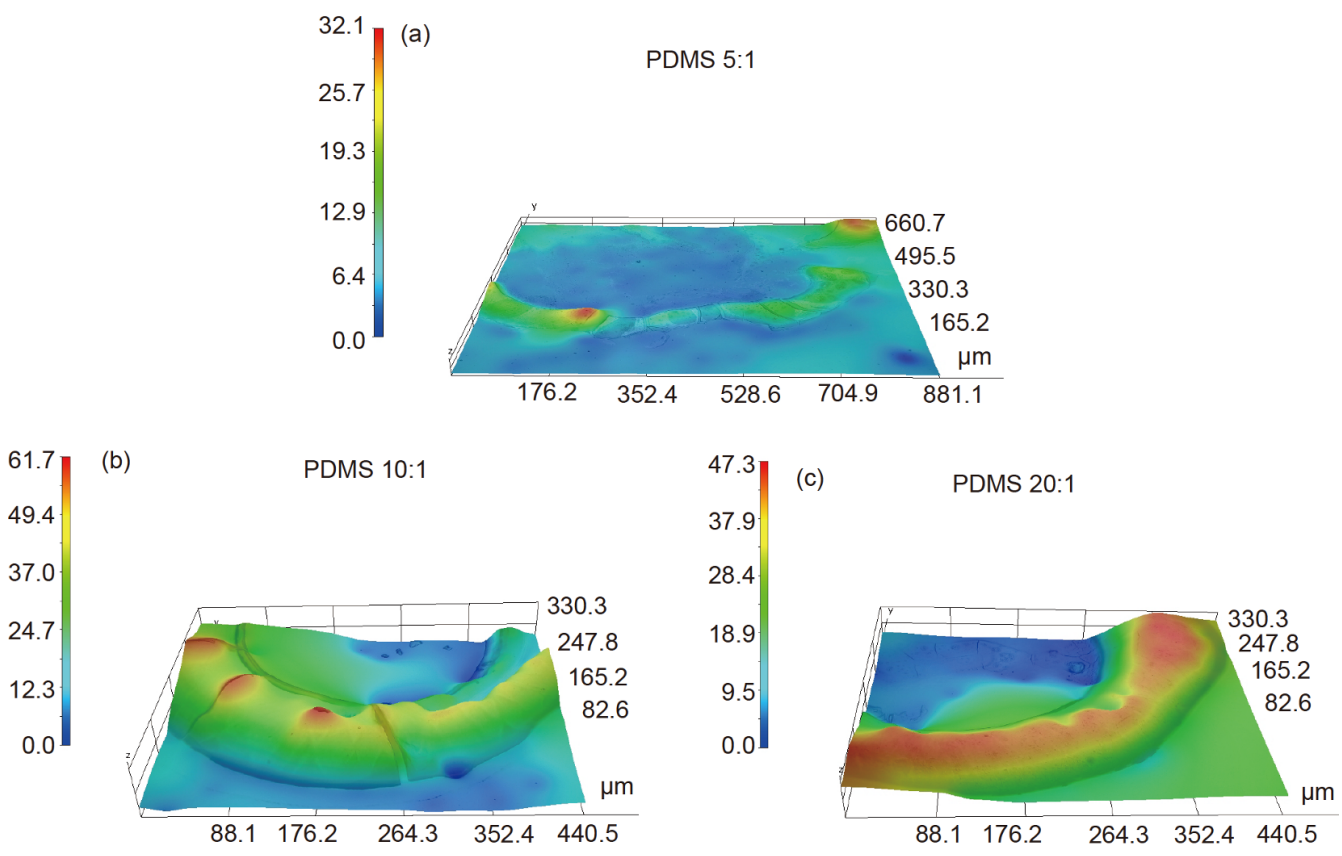

Figure 8 (Color online) Deposition profile of nanoparticles on PDMS surfaces. (a) PDMS 5:1; (b) PDMS 10:1; (c) PDMS 20:1.
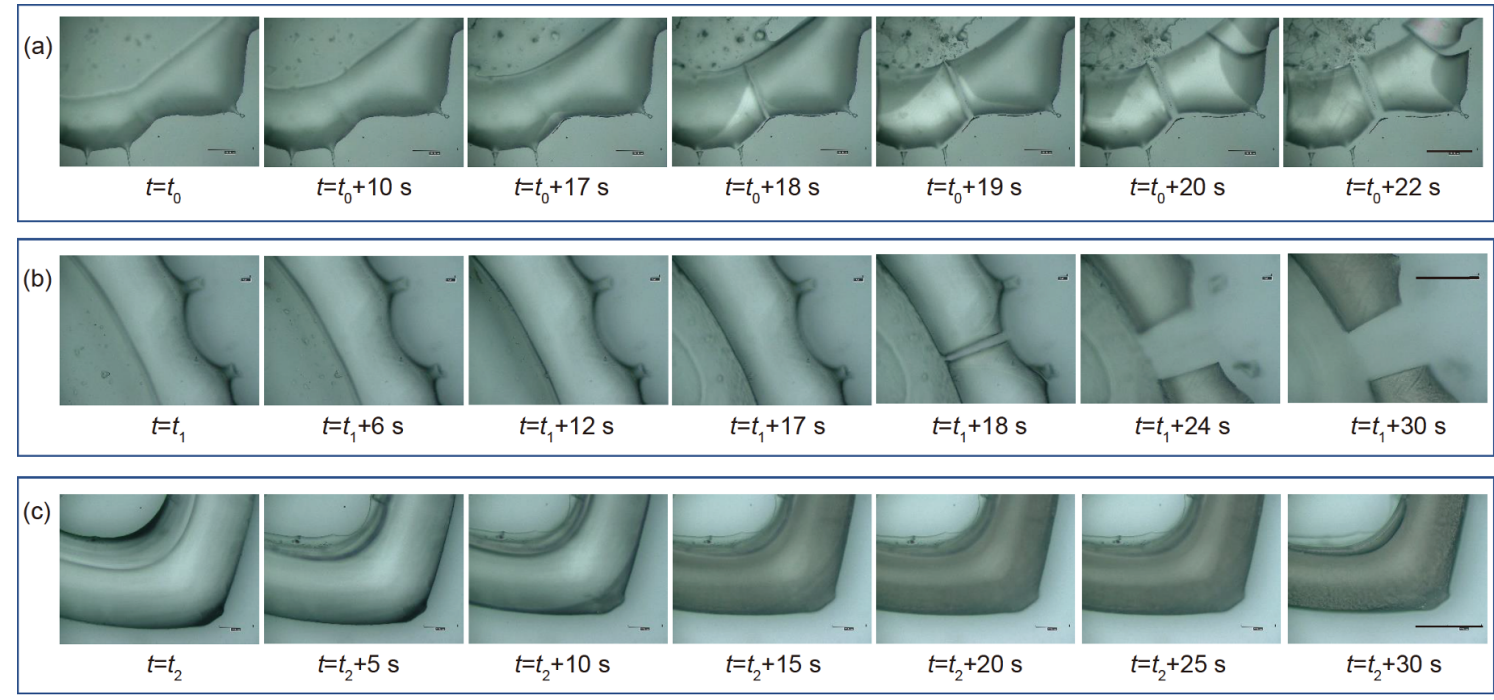

Figure 9 (Color online) Evolution of nanoparticle deposition on PDMS surfaces. (a) PDMS 5:1; (b) PDMS 10:1; (c) PDMS 20:1. Scale bars: 100 $\mu$ m.

the capillary force, the deposition would have to move until there was a new balance between them. This movement would produce a large fracture, otherwise clusters would be pinned and cracks would be formed as liquid bridges broke.

To quantitively determine the magnitude of the friction force, two kinds of forces (van der Waals force and electrostatic force) acting vertically in the final stage of evaporation of aqueous suspension with nanoparticles are considered. The van der Waals force between a nanoparticle and the substrate in water can be given as [46,51]:

$F_{\text {wps }}=\frac{A_{132} R}{6 z^{2}}$ where $A_{132}=\left(\sqrt{A_{11}}-\sqrt{A_{33}}\right)\left(\sqrt{A_{22}}-\sqrt{A_{33}}\right)$ with $A_{i i}$ denoting the Hamaker constant of material $i(i=1,2,3$ represents nanoparticle, substrate and water, respectively) [52], $R$ is the radius of the nanoparticle, and $z$ is the particlesubstrate separation distance.

The electrostatic force between a nanoparticle and the substrate in water is expressed as [46,51]:

$F_{\text {eps }}=-2 \varepsilon \kappa R \frac{\left[\phi_{1}^{2}+\phi_{2}^{2}-2 \phi_{1} \phi_{2} \exp (\kappa z)\right]}{[\exp (2 \kappa z)-1]}$,

where $\varepsilon$ and $\kappa$ are the permittivity of water and the reciprocal of Debye length, respectively, and $\phi_{i}(i=1,2)$ denotes the 
surface potential for material $i$. Neglecting nanoparticle gravity, the attractive force between a nanoparticle and substrate in water can be written as:

$F_{\mathrm{a}}=F_{\text {wps }}+F_{\text {eps }}$.

Then the friction force between a nanoparticle and the substrate in water can be given as:

$F_{\mathrm{f}}=f F_{\mathrm{a}}$,

where $f$ is the friction coefficient.

Table 1 lists all the parameters to calculate the above forces. As shown in Table 2, the electrostatic force between a nanoparticle and a silicon surface in water is nearly equal to that existing between a nanoparticle and PDMS surface in water due to the small difference in surface potential between PDMS and silicon. However, the Hamaker constant of silicon is much greater than that of PDMS, producing a van der Waals force between a PS nanoparticle and silicon in water more than 15 times greater than that of PS nanoparticle and
PDMS in water. Therefore, the vertical component of the interaction force between a PS nanoparticle and silicon is calculated to be $327.2 \mathrm{pN}$, approx. 17.42 times greater than that of a PS nanoparticle and PDMS (18.8 pN). Although the friction coefficients are not easily measured, the friction force between a PS nanoparticle and silicon in water will be much greater than that between a PS nanoparticle and PDMS in water. Moreover, both the Hamaker constant and surface potential of PDMS surface depend on the mass ratio of PDMS and should be studied further. Given the friction force between the PS nanoparticles and silicon is so great, clusters of nanoparticles are prevented from moving and radial cracks are formed. However, for the case of PDMS surfaces, the friction force becomes so low that nanoparticles have to move under the action of the capillary force until the balance is reached. Therefore, multiple large cracks are observed on PDMS surfaces.

Figure 10 shows the normalized volume loss versus time

Table 1 Parameters for calculation of interaction forces

\begin{tabular}{|c|c|c|c|}
\hline Symbol & Physical parameter & Value & Unit \\
\hline$A_{11}$ & Hamaker constant of PS nanoparticle & $6.3 \times 10^{-20}[53]$ & $\mathrm{J}$ \\
\hline$A_{22}^{(1)}$ & Hamaker constant of silicon & $20.60 \times 10^{-20}[54]$ & $\mathrm{J}$ \\
\hline$A_{22}^{(2)}$ & Hamaker constant of PDMS & $4.4 \times 10^{-20}[53]$ & $\mathrm{J}$ \\
\hline$A_{33}$ & Hamaker constant of water & $3.7 \times 10^{-20}[55]$ & $\mathrm{J}$ \\
\hline$A_{132}^{(1)}$ & $\begin{array}{l}\text { Hamaker constant between PS nano- } \\
\text { particle and silicon in water }\end{array}$ & $1.53 \times 10^{-20}$ & $\mathrm{~J}$ \\
\hline$A_{132}^{(2)}$ & $\begin{array}{l}\text { Hamaker constant between PS nano- } \\
\text { particle and PDMS in water }\end{array}$ & $0.10 \times 10^{-20}$ & $\mathrm{~J}$ \\
\hline$z$ & minimum separation distance & $0.4 \times 10^{-9}[51]$ & $\mathrm{m}$ \\
\hline$\varepsilon$ & permittivity of water & $7 \times 10^{-10}$ & $\mathrm{~F} / \mathrm{m}$ \\
\hline$\phi_{1}$ & surface potential of PS nanoparticle & $-59.5[56]$ & $\mathrm{mV}$ \\
\hline$\phi_{2}^{(1)}$ & surface potential of silicon & $-50[57]$ & $\mathrm{mV}$ \\
\hline$\phi_{2}^{(2)}$ & surface potential of PDMS & $-45[58]$ & $\mathrm{mV}$ \\
\hline$\kappa$ & reciprocal of the Debye length & $\left(430 \times 10^{-9}\right)^{-1}$ & $\mathrm{~m}^{-1}$ \\
\hline
\end{tabular}

Table 2 Values of interaction forces

\begin{tabular}{|c|c|c|c|}
\hline Symbol & Physical parameter & Value & Unit \\
\hline$F_{\text {wps }}^{(1)}$ & $\begin{array}{l}\text { van der Waals force between a PS } \\
\text { nanoparticle and silicon in water }\end{array}$ & 334.7 & $\mathrm{pN}$ \\
\hline$F_{\text {wps }}^{(2)}$ & $\begin{array}{l}\text { van der Waals force between a PS } \\
\text { nanoparticle and PDMS in water }\end{array}$ & 21.9 & $\mathrm{pN}$ \\
\hline$F_{\mathrm{eps}}^{(1)}$ & $\begin{array}{l}\text { electrostatic force between a PS } \\
\text { nanoparticle and silicon in water }\end{array}$ & -7.5 & $\mathrm{pN}$ \\
\hline$F_{\mathrm{eps}}^{(2)}$ & $\begin{array}{l}\text { electrostatic force between a PS } \\
\text { nanoparticle and PDMS in water }\end{array}$ & -3.1 & $\mathrm{pN}$ \\
\hline$F_{\mathrm{a}}^{(1)}$ & $\begin{array}{l}\text { vertical component of interaction force } \\
\text { between a PS nanoparticle and silicon } \\
\text { in water }\end{array}$ & 327.2 & $\mathrm{pN}$ \\
\hline$F_{\mathrm{a}}^{(2)}$ & $\begin{array}{c}\text { vertical component of interaction force } \\
\text { between a PS nanoparticle and PDMS } \\
\text { in water }\end{array}$ & 18.8 & $\mathrm{pN}$ \\
\hline
\end{tabular}




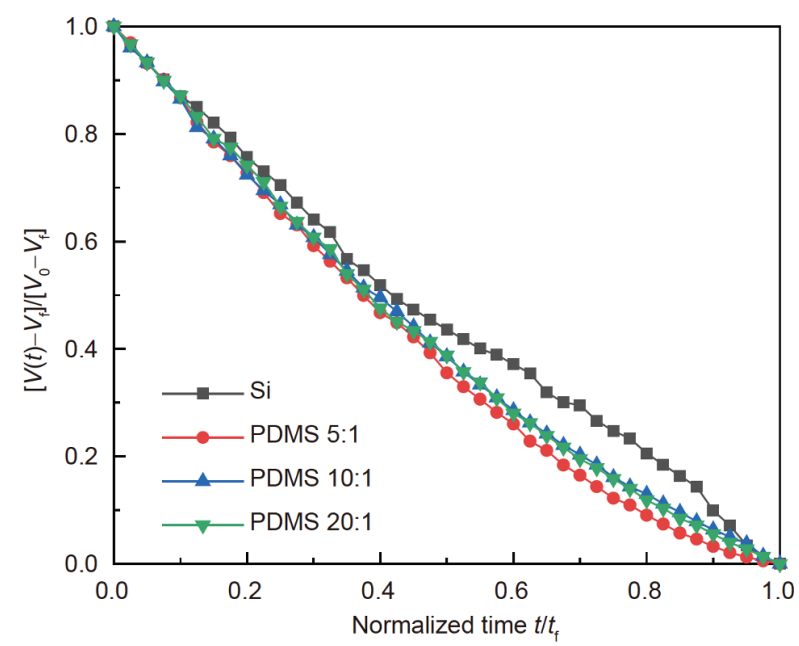

Figure 10 (Color online) Normalized volume variation of aqueous droplets on silicon and PDMS surfaces.

$\left(V_{0}, V(t)\right.$ and $V_{\mathrm{f}}$ are the initial volume, the instantaneous volume and the volume measured at the end of the final stage, respectively). Since the experimental temperature and relative humidity were held constant, the normalized volume variation is of the same order for different substrates. It should be noted that besides substrate elasticity, there are a lot of parameters including evaporation rate [59] and particle concentration which also play significant roles in the formation of cracks.

\section{Conclusions}

Evaporation of aqueous droplets containing $0.64 \mathrm{wt} \%$ PS nanoparticles on both silicon and PDMS surfaces was studied. It was found that CCR mode dominated the evaporation on clean silicon surface and the mass ratio of PDMS had a great influence on CCR duration. Radial cracks with periodic distribution were formed in the deposition of nanoparticles on silicon, while one to several large cracks were found on PDMS depending on its mass ratio. Detailed observation of crack nucleation demonstrated that the friction force between surface nanoparticles and the substrate played an important role in the formation of cracks.

This work was supported by the National Natural Science Foundation of China (Grant Nos. 11572114, and 11702299), the Chinese Academy of Sciences Strategic Priority Research Program (Grant No. XDB22040401), and PetroChina Innovation Foundation.

1 D. Bonn, J. Eggers, J. Indekeu, J. Meunier, and E. Rolley, Rev. Mod. Phys. 81, 739 (2009).

2 Z. Q. Lin. Evaporative Self-assembly of Ordered Complex Structures (World Scientific Publishing Co. Pte. Ltd., Singapore, 2012).

3 Y. P. Zhao. Physical Mechanics of Surfaces and Interfaces (Science Press, Beijing, 2012).

4 Y. P. Zhao. Nano and Mesoscopic Mechanics (Science Press, Beijing, 2014).
5 V. Dugas, J. Broutin, and E. Souteyrand, Langmuir 21, 9130 (2005).

6 D. Zang, S. Tarafdar, Y. Y. Tarasevich, M. Dutta Choudhury, and T. Dutta, Phys. Rep. 804, 1 (2019).

7 Z. P. Xu, and Q. S. Zheng, Sci. China-Phys. Mech. Astron. 61, 074601 (2018).

8 W. Yang, H. T. Wang, T. F. Li, and S. X. Qu, Sci. China-Phys. Mech. Astron. 62, 014601 (2018).

9 P. J. Yunker, T. Still, M. A. Lohr, and A. G. Yodh, Nature 476, 308 (2011).

10 H. H. Lee, S. C. Fu, C. Y. Tso, and C. Y. H. Chao, Int. J. Heat Mass Transfer 105, 230 (2017).

11 Y. S. Yu, M. C. Wang, Y. Q. Zhu, J. Z. Zhou, and A. Zhou, Chem. Eng. Sci. 205, 212 (2019).

12 M. Parsa, S. Harmand, and K. Sefiane, Adv. Colloid Interface Sci. 254, 22 (2018).

13 Y. Li, C. Lv, Z. Li, D. Quéré, and Q. Zheng, Soft Matter 11, 4669 (2015).

14 N. D. Patil, P. G. Bange, R. Bhardwaj, and A. Sharma, Langmuir 32, 11958 (2016).

15 V. X. Nguyen, and K. J. Stebe, Phys. Rev. Lett. 88, 164501 (2002).

16 U. U. Ghosh, M. Chakraborty, S. De, S. Chakraborty, and S. DasGupta, Langmuir 32, 12790 (2016).

17 W. Sun, and F. Yang, J. Phys. Chem. C 118, 10177 (2014).

18 S. Zhang, W. Luan, Q. Zhong, S. Yin, and F. Yang, Soft Matter 12, 8285 (2016).

19 D. Mampallil, H. B. Eral, D. van den Ende, and F. Mugele, Soft Matter 8, 10614 (2012).

20 M. Majumder, C. S. Rendall, J. A. Eukel, J. Y. L. Wang, N. Behabtu, C. L. Pint, T. Y. Liu, A. W. Orbaek, F. Mirri, J. Nam, A. R. Barron, R. H. Hauge, H. K. Schmidt, and M. Pasquali, J. Phys. Chem. B 116, 6536 (2012).

21 W. Li, D. Lan, H. Sun, and Y. Wang, Langmuir 34, 4715 (2018).

22 R. D. Deegan, O. Bakajin, T. F. Dupont, G. Huber, S. R. Nagel, and T. A. Witten, Nature 389, 827 (1997).

23 H. Hu, and R. G. Larson, J. Phys. Chem. B 110, 7090 (2006).

24 E. R. Dufresne, E. I. Corwin, N. A. Greenblatt, J. Ashmore, D. Y. Wang, A. D. Dinsmore, J. X. Cheng, X. S. Xie, J. W. Hutchinson, and D. A. Weitz, Phys. Rev. Lett. 91, 224501 (2003).

25 K. B. Singh, and M. S. Tirumkudulu, Phys. Rev. Lett. 98, 218302 (2007).

26 L. Pauchard, B. Abou, and K. Sekimoto, Langmuir 25, 6672 (2009).

27 Y. Zhang, Y. Qian, Z. Liu, Z. Li, and D. Zang, Eur. Phys. J. E 37, 84 (2014).

28 C. C. Annarelli, J. Fornazero, J. Bert, and J. Colombani, Eur. Phys. J. E 5, 599 (2001).

29 M. N. Gao, X. F. Huang, and Y. P. Zhao, Sci. China Technol. Sci. 61, 949 (2018).

30 D. Brutin, B. Sobac, B. Loquet, and J. Sampol, J. Fluid Mech. 667, 85 (2011).

31 L. Pauchard, M. Adda-Bedia, C. Allain, and Y. Couder, Phys. Rev. E 67, 027103 (2003).

32 G. Jing, and J. Ma, J. Phys. Chem. B 116, 6225 (2012).

33 L. Goehring, W. J. Clegg, and A. F. Routh, Soft Matter 7, 7984 (2011).

34 F. Carle, and D. Brutin, Langmuir 29, 9962 (2013).

35 V. Lazarus, and L. Pauchard, Soft Matter 7, 2552 (2011).

36 Q. Jin, P. Tan, A. B. Schofield, and L. Xu, Eur. Phys. J. E 36, 28 (2013).

37 R. Pericet-Camara, A. Best, H. J. Butt, and E. Bonaccurso, Langmuir 24, 10565 (2008).

38 R. W. Style, R. Boltyanskiy, Y. Che, J. S. Wettlaufer, L. A. Wilen, and E. R. Dufresne, Phys. Rev. Lett. 110, 066103 (2013).

39 Y. S. Yu, and Y. P. Zhao, J. Colloid Interface Sci. 339, 489 (2009).

40 Y. Yu, Appl. Math. Mech.-Engl. Ed. 33, 1095 (2012).

41 L. Chen, E. Bonaccurso, T. Gambaryan-Roisman, V. Starov, N. Koursari, and Y. Zhao, Curr. Opin. Colloid Interface Sci. 36, 46 (2018).

42 M. C. Lopes, and E. Bonaccurso, Soft Matter 8, 7875 (2012). 
43 Y. S. Yu, Z. Q. Wang, and Y. P. Zhao, Acta Mech. Sin. 29, 799 (2013).

44 J. Gerber, T. Lendenmann, H. Eghlidi, T. M. Schutzius, and D. Poulikakos, Nat. Commun. 10, 4776 (2019).

45 Y. Chen, A. Askounis, V. Koutsos, P. Valluri, Y. Takata, S. K. Wilson, and K. Sefiane, Langmuir 36, 204 (2020).

46 Y. S. Yu, X. L. Xia, X. Zheng, X. Huang, and J. Z. Zhou, Sci. ChinaPhys. Mech. Astron. 60, 094612 (2017).

47 Y. S. Yu, M. C. Wang, and X. F. Huang. Sci. Rep. 7, 14118 (2017).

48 M. C. Lopes, and E. Bonaccurso, Soft Matter 9, 7942 (2013).

49 W. Dai, and Y. P. Zhao, Int. J. Nonlin. Sci. Numer. Simul. 8, 579 (2007).

50 S. Dash, A. Chandramohan, and S. V. Garimella, J. Heat Transfer 136, 080917 (2014).
51 T. S. Wong, T. H. Chen, X. Shen, and C. M. Ho, Anal. Chem. 83, 1871 (2011).

52 Y. P. Zhao, Sci. China Tech. Sci. 62, 2310 (2019).

53 C. J. Drummond, and D. Y. C. Chan, Langmuir 13, 3890 (1997).

54 A. Reindl, and W. Peukert, J. Colloid Interface Sci. 325, 173 (2008).

55 A. Koh, G. Gillies, J. Gore, and B. R. Saunders, J. Colloid Interface Sci. 227, 390 (2000).

56 A. Nakao, Y. Suzuki, and M. Iwaki, J. Colloid Interface Sci. 197, 257 (1998).

57 J. Binner, and Y. Zhang, J. Mater. Sci. Lett. 20, 123 (2001).

58 B. J. Kirby, and E. F. Hasselbrink, Electrophoresis 25, 203 (2004).

59 K. Piroird, V. Lazarus, G. Gauthier, A. Lesaine, D. Bonamy, and C. L. Rountree, Eurphys. Lett. 113, 38002 (2016). 